\title{
Premiers résultats dans l'infestation par Strongyloides stercoralis de la souris traitée par le Cyclophosphamide
}

\author{
par Geneviève MOUGEOT et Félix LANCASTRE \\ et la collaboration technique de J. Bolognini et C. Dubois \\ Laboratoire de Parasitologie de la Faculté de Médecine Saint-Antoine ( $\mathrm{P}^{\mathrm{r}}$ Golvan), \\ 27, rue Chaligny, F 75 - Paris $\left(12^{\circ}\right)$
}

\begin{abstract}
Résumé
Chez des souris recevant quotidiennement pendant 6 jours en injection intrapéritonéale une substance immunosuppressive (Cyclophosphamide) (1), les auteurs ont pu obtenir l'infestation par Strongyloides stercoralis, l'Anguillule de l'Homme.
\end{abstract}

\section{Summary}

In white mice daily injected for six days, with immunosuppressive drug (Cyclophosphamide), the infection by Strongyloides siercoralis, the Human Strongyloides, has been obtained by the authors.

Les différentes méthodes utilisées en Parasitologie et permettant de rendre la Souris immunotolérante poursuivent un double but.

$1^{\circ}$ Obtenir chez un animal le maintien ou le cycle de parasites réputés strictement humains.

(1) Endoxan-Asta. Laboratoires A. Lucien. 
$2^{\circ}$ Permettre l'étude au laboratoire du cycle ou des parties du cycle parasitaire, y compris la maturation sexuelle.

Dans cette optique, de nombreuses techniques ont été mises au point :

- Utilisation de la cortisone par Galliard et Berdonneau (1953), Olson (1959) et Parker (1960).

- Emploi des hormones sexuelles dès 1939 par Clapham.

Nous avons décrit en 1968 une première méthode qui consistait à obtenir, par injection massive de blanc d'œuf dans la cavité péritonéale de souris, la saturation antigénétique de tout le système immunitaire de l'animal-hôte. (Bazin et Lancastre).

Grâce à cette méthode, des larves infestantes de Necator americanus ont pu évoluer chez la souris jusqu'au stade adulte sexuellement bien différencié, mais sans production d'œufs ou de larves (Lancastre et coll., 1969). De même, des larves au stade IV de Trichinella spiralis injectées dans la cavité péritonéale ont pu acquérir des caractères sexuels nets, et l'injection intracoecale de formes végétatives d'Entamoeba hystolytica a permis d'obtenir chez les animaux traités des formes hématophages. (Lancastre et coll., 1968 et 1969).

La deuxième méthode utilisée, consiste en l'injection intrapéritonéale de phosphamide cyclique de moutarde azotée ou cyclophosphamide (Endoxan Asta) *. Cytostatique et cytotoxique, ce produit a une action dépressive sur le système leucopoiétique - et par conséquent sur la production d'anticorps.

\section{Protocole expérimental.}

Des lots de souris reçoivent quotidiennement, pendant 6 jours, en injection intrapéritonéale une dose de l'ordre de $200 \mathrm{mg} / \mathrm{kg}$, soit $30 \mathrm{mg}$ de produit par cure.

Le deuxième jour après la fin des injections, les souris sont infestées par voie transcutanée avec des larves infestantes de Strongyloides stercoralis obtenues par coprocultures sur sciure de bois en boîte de Petri. Ces coprocultures sont faites avec des selles de sujets originaires des Antilles, reconnus infestés à la consultation de Pathologie parasitaire et tropicale de l'hôpital Saint-Antoine ( $\mathrm{P}^{r}$ Golvan).

L'infestation est massive (2.000 à 3.000 larves par souris) et la durée du bain infestant est de 2 heures environ.

\section{Résultats.}

Au cours de la première semaine suivant l'infestation, la mortalité est importante (30\% des souris). 
Au $15^{\circ}$ jour de l'infestation, une partie des animaux est autopsiée. Le duodénum et l'intestin grêle sont recueillis dans une boîte de Petri contenant du sérum physiologique tiédi. Macroscopiquement, le duodénum apparaît dilaté ; il existe même des zones hémorragiques. Après ouverture et examen à la loupe binoculaire du duodénum et de la partie haute du jéjunum, des adultes (jeunes femelles parthénogénétiques de Strongoloïdes stercoralis (sont récupérés (fig. 1). On a constaté chez une souris la présence de 8 vers.

$\mathrm{Au} 20^{\circ}$ jour de l'infestation, d'autres souris sont sacrifiées. Le duodénum et le jéjunum sont recueillis comme précédemment. Ces organes apparaissent également dilatés et hyperhémiés; on y constate la présence de quelques femelles parthénogénétiques adultes, ainsi que d'œufs embryonnés, enchâssés dans la muqueuse (fig. 2). On observe de nombreuses larves rhabditoïdes nageant dans le contenu de la boîte de Petri (fig. 3).

\begin{tabular}{|c|c|}
\hline $\begin{array}{l}\text { Strongyloides } \\
\text { stercolaris }\end{array}$ & Dimensions \\
\hline Femelles parthénogénétiques adultes $\ldots \ldots \ldots \ldots$ & 1,7 à $1,5 \mathrm{~mm}$ \\
\hline 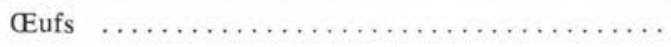 & $70-54 \mu / 34-38 \mu$ \\
\hline Larves $\ldots \ldots \ldots \ldots$ & $150-120 \mu / 12-15 \mu$ \\
\hline
\end{tabular}

Tous les animaux restants furent sacrifiés et autopsiés le $24^{e}$ jour. Aucun d'entre eux n'était parasité. On peut supposer qu'ils ont échappé à l'infestation, ou encore, ce qui paraît plus vraisemblablé, qu'ils se sont déparasités spontanément, l'effet immunosuppresseur ayant cessé.

\section{Conclusion.}

Il semble que dans les relations hôte-parasite, comme dans la «spécificité parasitaire », le système immunologique de l'hôte intervienne, et qu'une inertie de ce système tende à favoriser l'adaptation de parasites normalement inféodés à l'homme, ou tout au moins, de certains d'entre eux.

Les premiers résultats obtenus jusqu'ici dans l'emploi du cyclophosphamide nous permettent d'évaluer la durée de l'effet d'une série unique d'injections à environ 15-20 jours. Nos recherches actuelles tendent au maintien de l'infestation dans le but de réaliser une strongyloïdose analogue à celle de l'homme. 


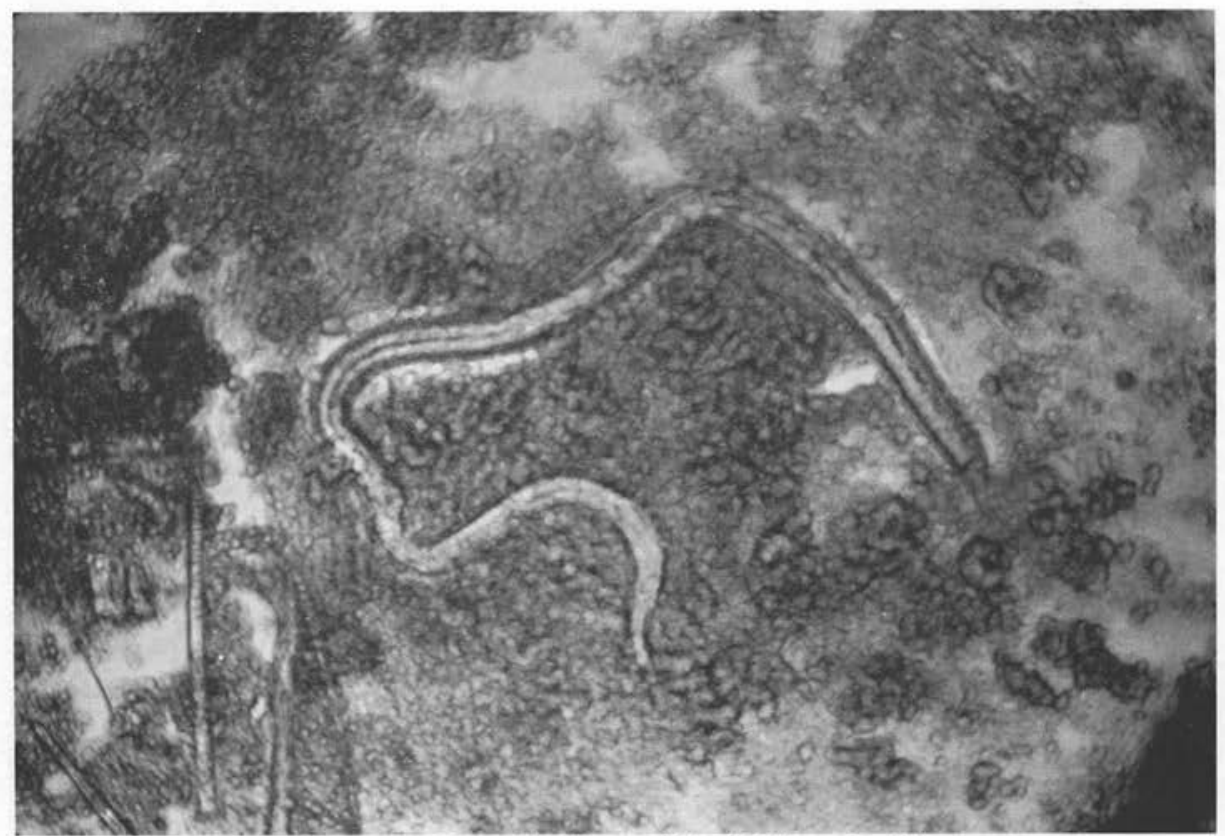

Fig. 1. - Jeune femelle parthénogénétique $(\times 10)$.

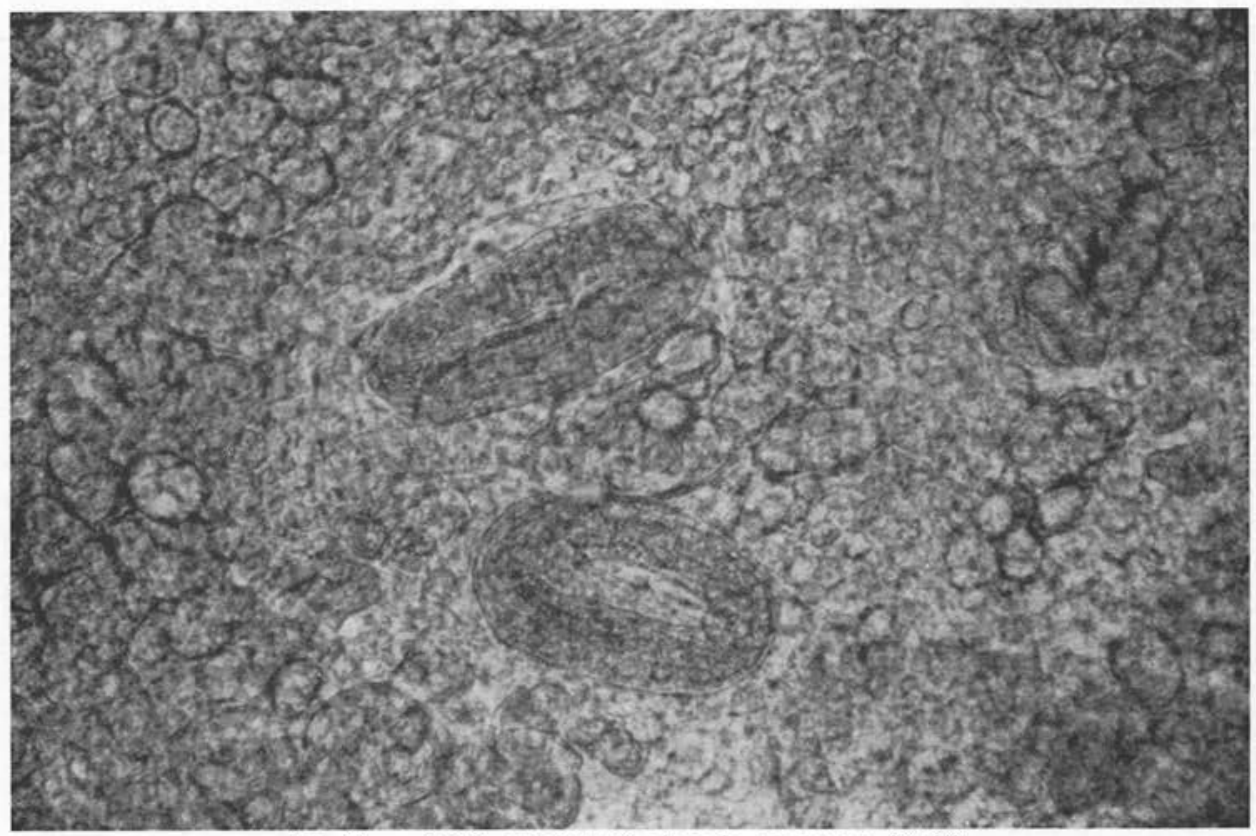

Fig. 2. - Eufs embryonnés dans la muqueuse $(\times 40)$. 


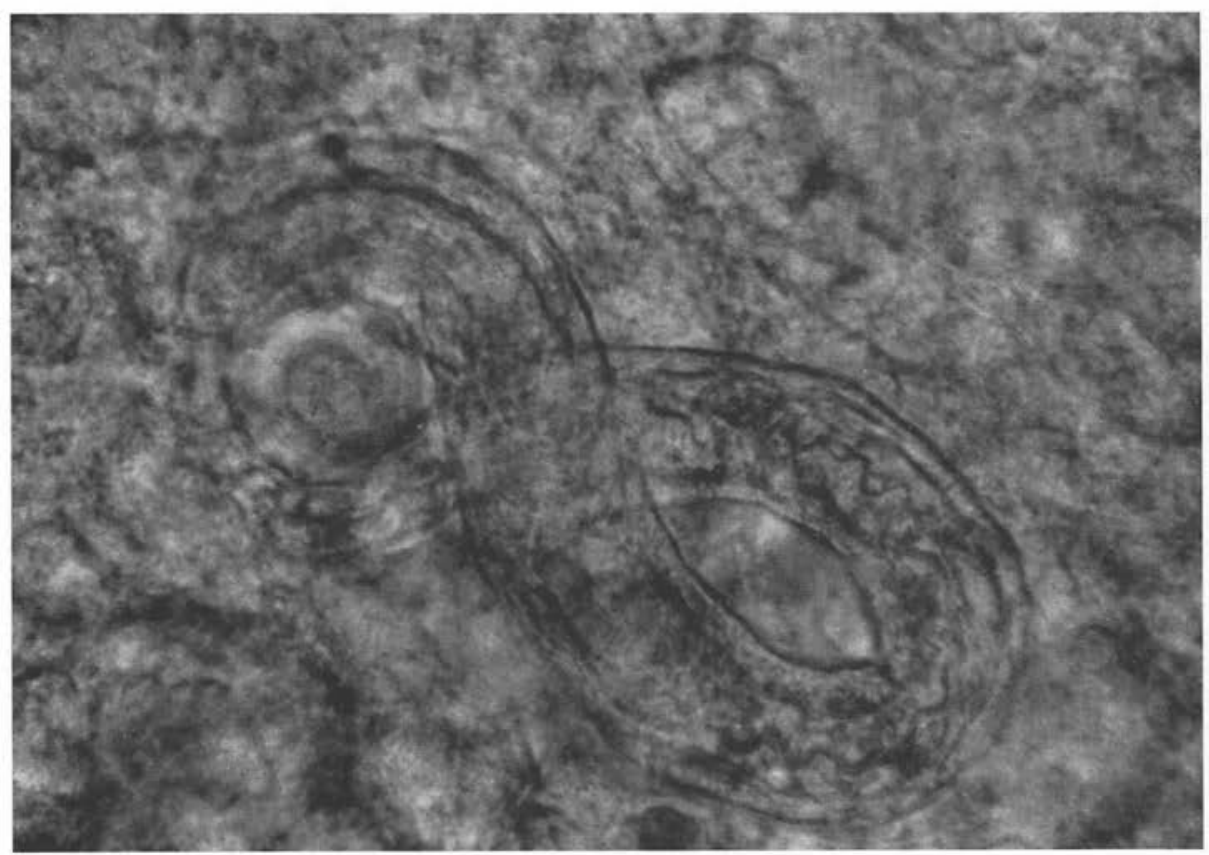

Fig. 3. - Larve rhabditoide (immersion).

\section{Bibliographie}

Bazin (J.-C.), LANCASTRE (F.), 1968. - L'utilisation en parasitologie de souris rendues immunotolérantes par injection de protéines hétérologues. Annales parasit. hum. et comp. (Paris), 43, 718.

Clapham (P. A.), 1939. - On a sex difference in the infection rate of birds with Syngamus trachea. J. Helminth., 17, 192-194.

Galliard (H.) et Berdonneau (R.), 1953. - Strongyloïdose expérimentale chez le Chien. Effet de la cortisone. Résultats du test de Thorn à l'hormone corticotrope (A.C.T.H.). Annales Parasit., 28, 163-171.

Lancastre (F.), Bazin (J.-C.), Gargouri (M.), Le Fichoux (Y.), Mougeot (G.), 1968. Amibiase expérimentale. Annales Parasit. hum. comp. (Paris), 43, 623-628.

-, 一, Golvan (Y. J.), Deltour (F.), Mougeot (G.), Houin (R.), 1969. - Infestation par Necator americanus de Souris blanches rendues immunotolérantes par injections massives de protéines hétérologues. Annales parasit. hum. comp. (Paris), 44, 241.

—, - 1969. - Survie de divers stades de Trichinella spiralis dans la cavité péritonéale de la souris (normale et immunotolérante). Proceedings of the second International Conference on Trichinellosis Wroclaw, June 26-28 1969. 
—, Mougeot (G.), 1970. - Obtention de femelles parthénogénétiques dans l'infestation par Strongylö̈des stercoralis de la souris préalablement traitée par le Cyclophosphamide. Société française de Parasitologie (Lyon), 9-10 mai 1970.

OLson (L.-J.), 1959. - The cellular response of white Rats to Litomosö̈des carinni larvae as influenced by cortisone age and previous infection. J. Parasit., 45, 519-532.

PARKer (J.-C.), 1960. - Effect of cortisone on the resistance of the Guinea pig to infection with the Rat nematode Nipponstrongylus brasiliensis. Exptl. Parasit., 11, 380-390. 